PERCEPCIÓN DEL ÉXITO EMPRESARIAL, DESDE LA PERSPECTIVA DE PLANEACIÓN EN EL MICROEMPRESARIO EN PUEBLA.

\title{
PERCEPCIÓN DEL ÉXITO EMPRESARIAL, DESDE LA PERSPECTIVA DE PLANEACIÓN EN EL MICROEMPRESARIO EN PUEBLA
}

\section{PERCEPTIONS ON ENTREPRENEURIAL SUCCESS, FROM THE PLANNING PERSPECTIVE OF THE MICROENTREPRENEUR IN PUEBLA}

\author{
Edgar Flores Bautista*
}

*Maestro en Administración. Docente de la Facultad de Administración. Universidad Autónoma de Puebla. ORCID: https://orcid.org/0000-0003-2155-3977.

Dirección para recibir correspondencia: flores_edgar@hotmail.com

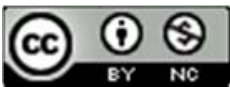


PERCEPCIÓN DEL ÉXITO EMPRESARIAL, DESDE LA PERSPECTIVA DE PLANEACIÓN EN EL MICROEMPRESARIO EN PUEBLA.

\section{RESUMEN}

OBJETIVO: Identificar la percepción que tienen los empresarios de la ciudad de Puebla sobre los factores de éxito empresarial en las microempresas, dedicadas a la compra-venta de artículos de consumo básico del centro de la ciudad de Puebla.

MATERIAL Y MÉTODO: Se trata de una investigación de tipo concluyente, descriptiva y no experimental. La encuesta se aplicó a una muestra por conveniencia, integrada por 55 microempresas dedicadas a la compra-venta de artículos de consumo básico, como: mercerías, abarroteras, misceláneas, jugueterías y papelerías del centro de la ciudad de Puebla.

RESULTADOS: Se obtuvo una fiabilidad aceptable (0.782) y como resultados importantes, se tiene que el perfil del microempresario en un $47 \%$ de ellos son jóvenes entre 26 y 35 años. Quienes heredaron el negocio por parte de sus padres el $53 \%$ y sólo el $36 \%$ lo inició con sus propios recursos y esfuerzos. Asimismo, desde la percepción de planeación para la formulación y práctica de estrategias, los empresarios entrevistados consideran que el éxito de sus negocios se ha logrado a través de contar con fondos de ahorro para situaciones de crisis económica, la inversión en programas de capacitación de su personal; además de la implementación de tecnología (como lector de códigos de barras, software contable, uso de redes sociales) y la introducción de productos y servicios nuevos.

CONCLUSIONES: Como hallazgo se tiene, que el microempresario no cree en los apoyos y programas gubernamentales para la MiPyME, y argumenta que no ayuda en nada a la consolidación de sus negocios por burocráticos y por la complicación en la práctica empresarial. Se puede concluir que el microempresario, está consciente que la implementación de tecnología, gestión de recursos, utilizar publicidad y propaganda en sus negocios, ofrecer un servicio o producto con valor agregado, y la motivación a los empleados, puede diferenciarlos frente a la competencia y permanecer más tiempo logrando el éxito empresarial. Por ello, todavía el empresario mexicano, sigue viviendo un empirismo empresarial, pero con una creciente necesidad y claridad en formarse en estrategias de negocio para lograr que sus empresas sobrevivan, permanezcan y sean rentables.

PALABRAS CLAVE: MiPyME. Éxito empresarial. Negocios. 
PERCEPCIÓN DEL ÉXITO EMPRESARIAL, DESDE LA PERSPECTIVA DE PLANEACIÓN EN EL MICROEMPRESARIO EN PUEBLA.

\section{ABSTRACT}

OBJECTIVE: To identify the perception entrepreneurs have on entrepreneurial factors of success in microenterprises dedicated to the buying-selling of basic goods in downtown Puebla.

MATERIAL AND METHOD: This is a conclusive, descriptive and non-experimental research. The survey was administered to a convenient sample formed by 55 microenterprises, such as: miscellaneous or grocery stores, toy stores and stationary stores located in downtown Puebla, dedicated to the buying-selling of basic goods.

RESULTS: The acceptable reliability was 0.782 and as relevant results, the profile of the microentrepreneur obtained is that $47 \%$ of the participants is between 26 and 35 years old. $53 \%$ inherited the business from their parents and only a $36 \%$ started their businesses with own resources and efforts. From the planning perspective for the formulating and putting into practice of strategies, the participants consider that business success has been achieved through personal savings as an economic crisis backup plan, and investment in training programs for their personnel; technology implementation, such as: bar code scanners, accounting software, use of social media; and the introduction of new products and services.

CONCLUSIONS: The microentrepreneur does not believe in government programs or support for SME's and mentions that those programs do not really help businesses consolidations due to bureaucracy and complication of the entrepreneurial practice. It can be concluded that the microentrepreneur is aware of technological implementation, resource management, promotion, added value in products or services and employees' motivation. The entrepreneur has the ability to discriminate them and maintain them through time achieving entrepreneurial success. Opposite to the Mexican entrepreneur who is still living in an entrepreneurial empirical knowledge but with a latent need to continue learning business strategies in order to have prevailing and profitable companies.

KEYWORDS: MiPyME. Business success. Business. 
PERCEPCIÓN DEL ÉXITO EMPRESARIAL, DESDE LA PERSPECTIVA DE PLANEACIÓN EN EL MICROEMPRESARIO EN PUEBLA.

\section{ANTECEDENTES}

La Micro, Pequeña y Mediana Empresa (MiPyME), está concebida como una pequeña unidad socioeconómica que puede pertenecer al sector de producción, de comercio o de prestación de servicios; además puede o no contar con grandes inversiones de capital para su creación, ya que ahora la empresa puede ser virtual o con instalaciones físicas (Tinoco-Cantillo, Buelvas-Martínez y Buelvas-Meza, 2003). Las microempresas son importantes en la economía tanto en países desarrollados como en países de Latinoamérica, ya que constituyen en ambas situaciones entre el $70 \%$ y el $90 \%$ del total de las empresas. Las MiPyME se constituyen, hoy en día, en la economía como agentes dinámicos y diversos en América Latina y en una parte fundamental en términos de generación de empleo y desarrollo. En Latinoamérica, la MiPyME representa más del 96\% de las unidades económicas actuales, (Bautista y Benavides, 2013). Así, para el caso de México, más del $98 \%$ de las empresas son micro, pequeñas y medianas, y son fuentes generadoras de riqueza y empleo (INEGI, 2015).

En este trabajo, se describe un análisis de la situación actual de la MiPyME, permitiendo la identificación del valor intangible que presentan las empresas de este tamaño y proporcionará datos para comprender la dinámica empresarial, desde la planeación de las acciones que los empresarios han vivido para el éxito (permanencia) de sus negocios.

\section{Las MiPyME en la ciudad de Puebla}

El estado de Puebla representa el $5.2 \%$ de población del país, con un total de $6,178,883$ habitantes (INEGI, 2015). Aporta el 3.2\% del PIB nacional y el sector de actividad con que mayormente coopera está relacionado con el comercio y las unidades económicas en el Estado ascienden a 251,308 (INEGI, 2014), estando dentro de los seis Estados del país que generan mayor número de unidades económicas, específicamente la ciudad de Puebla genera 72,755, siendo estos el 55\% de establecimientos se dedican al comercio (Tello, Hernández y LariosGómez, 2018). En Puebla, la MiPyME representa el $99 \%$ de las empresas de la entidad y generan más de 459 mil empleos o $75.4 \%$ de los empleos en industria, comercio y manufactura y es el quinto estado con más MiPyME. 
PERCEPCIÓN DEL ÉXITO EMPRESARIAL, DESDE LA PERSPECTIVA DE PLANEACIÓN EN EL MICROEMPRESARIO EN PUEBLA.

\section{Planeación en la micro empresa}

La planeación en la empresa, es un proceso que permite formular y ejecutar acciones llamadas estrategias con la finalidad de lograr objetivos y metas para permanencia y supervivencia en el mercado competitivo (Larios-Gómez, 2016a). Independientemente que la planeación sea vista como elemento básico del proceso administrativo (Chiavenato, 2011), en la MiPyME se debe de entender como una herramienta de visión global, permite realizar toma de decisiones de profundo análisis del entorno y por ende tomar decisiones con visión y certeza del mundo global (Moreno, Moreno y Dávalos, 2015). Según Drucker (2002), la planeación desde una visión estratégica, se puede definir como el proceso continuo, basado en una perspectiva posible del futuro, a través de la toma de decisiones en el presente. Teniendo implicaciones en los resultados esperados, que se medirán sistemáticamente frente a las expectativas que se hayan generado. Para este caso, la planeación en la MiPyME, se trata de las acciones que visualiza a futuro el empresario a partir de sus experiencias en el negocio, que involucra factores internos y externos del mercado donde impacta, para proyectar un futuro de certidumbre que le permita la permanencia y el éxito empresarial (Larios-Gómez, 2016a).

En la planeación empresarial, existen factores internos o externos, que permiten la permanencia de las microempresas a través de un lapso prolongado en el tiempo, que pueden ser parecidos o diferentes para cada tipo y tamaño de empresa, pero a pesar de esto surge la inquietud por conocer cuáles son las condiciones o aquello que les ha permitido a las microempresas, haberse consolidado o permanecido en el mercado, (Martín, 2013). Cómo dar paso a la apertura del análisis de aquellos factores que son determinantes para conseguir el éxito de una microempresa, y al mismo tiempo, darle la importancia del papel que desempeñan y los beneficios que aportan a la sociedad con el fin de mantenerlas vigentes o competitivas como empresas. En una investigación realizada por Larios-Gómez (2016b, p. 112) señala que los elementos de planeación que influyen en la competitividad de las empresas micro, pequeñas y medianas son:

“1. La gestión de los flujos de producción (materias primas e inventarios), 2. La gestión de los mecanismos de interacción entre planeación, mercadotecnia, investigación y el desarrollo formal, diseño, ingeniería en la producción industrial, 3. La capacidad para combinar actividades de investigación y desarrollo e innovación en cooperación con universidades y otras empresas, 4 . La capacidad para incorporar definiciones más exactas de características de la demanda y evolución de mercados en el diseño y producción de FLORES-BAUTISTA E. 
PERCEPCIÓN DEL ÉXITO EMPRESARIAL, DESDE LA PERSPECTIVA DE PLANEACIÓN EN EL MICROEMPRESARIO EN PUEBLA.

estrategias, 5. La capacidad para organizar con éxito relaciones inter-empresariales con proveedores y clientes y la mejora de las capacidades de los trabajadores a través de la inversión en entrenamiento especializado y en la generación de altos niveles de responsabilidad en los trabajadores de producción".

\section{Problema}

Hablar sobre la supervivencia de la MiPyME, es determinar las acciones a las que se ven enfrentadas ante la presencia de las competencias o ante la misma decisión del consumidor, y de identificar las diferentes debilidades de carácter estructural que presenta la empresa, que son las debilidades intrínsecas al tamaño de las empresas. Arias Sandoval y Quiroga Marín (2008), observa tres determinantes o puntos de influencia en la supervivencia empresarial, los factores internos propios de la empresa (estructura, capacidad, el capital humano), los factores externos propios al entorno (competencia, economía, tendencias de consumo) y los factores de conocimiento empresarial (perfil del empresario, educación empresarial). Estos factores como posibles causas que muestren el cierre de las empresas en sus primeros años de funcionamiento, donde la supervivencia se ve influenciada por estos factores y el tamaño de la misma empresa (Medina y de Lema, 2012).

Parra (2011), analiza la probabilidad de cierre de la MiPyME antes de alcanzar los primeros cinco años de funcionamiento, debido a que las condiciones macroeconómicas y microeconómicas y su relación con el sector al que pertenece, afectan directamente el financiamiento, la capacidad de innovación, y mercadeo y publicidad de la empresa. Moreno, Moreno y Dávalos (2015) en una comparación de los tiempos de funcionamiento de las empresas y los factores que influyen en dicho tiempo de permanencia, determina que en el ámbito de la economía industrial es de gran importancia tener conocimiento de lo que sucede en las empresas una vez entran al mercado, dado que los efectos de la movilidad empresarial sobre los diferentes sectores de la industria no dependen solamente de los procesos de entrada y salida de empresas, sino también de sus procesos de expansión.

Pérez, Martínez, De Luis y Vela (2006) confirman que las empresas jóvenes fracasan debido a las deficiencias que estas presentan en los conocimientos de gestión financiera y las empresas con mayor trascendencia en el mercado tienen la probabilidad de quebrarse esto debido a la poca capacidad de adaptación a los cambios del mercado. Sostienen que la edad de estas empresas FLORES-BAUTISTA E. 
PERCEPCIÓN DEL ÉXITO EMPRESARIAL, DESDE LA PERSPECTIVA DE PLANEACIÓN EN EL MICROEMPRESARIO EN PUEBLA.

está relacionada con la probabilidad de supervivencia o fracaso de la compañía y además que el fracaso se vuelve más probable en el caso de desalineación entre lo que la empresa puede hacer y lo que el entorno competitivo requiere. Estrada y Dutrénit (2009), en un estudio donde profundizan acerca del ciclo de vida de la empresa, determinan que desde el proceso que representa el surgimiento y cierre de las empresas en el tiempo, está involucrada la estrategia de creación y desarrollo de nuevos productos o mejoras innovadoras.

La ciudad de Puebla, a pesar de tener una posición geográfica privilegiada para su desarrollo económico y crecimiento empresarial. Del total de la MiPyME dedicadas al sector comercial, en promedio el $60 \%$ de estas empresas no sobreviven más de 5 años en el mercado (Ortiz, Rodríguez \& Izquierdo, 2013), como se ha explicado en apartados anteriores con resultados de investigaciones respecto a la competitividad y diagnóstico de la MiPyME, esto se debe a varios factores internos, externos y de la capacidad empresarial de los dueños de las empresas. Entonces, para el caso de un grupo de empresas micro del sector comercial en la ciudad de Puebla, ¿Cuáles son los factores de planeación determinantes para alcanzar el éxito de las microempresas del sector comercial/servicios en la ciudad de Puebla? ¿Cuál es el perfil del microempresario en Puebla? ¿Cuáles son los factores que mayor influencia tienen sobre los microempresarios poblanos para alcanzar el éxito? ¿Identifica el microempresario los factores de planeación que ayudan al crecimiento de su negocio?

\section{MATERIAL Y MÉTODO}

La presente investigación es de tipo concluyente, descriptiva y no experimental, debido a que es una investigación que pretende describir el perfil del empresario micro y que éste pueda tomar decisiones con los resultados presentados, además de que no se manipularon las variables de estudio (Kerlinger, Lee, Pineda y Mora, 2002). Teniendo como objetivo, identificar la percepción de los factores de planeación de éxito empresarial en los microempresarios de la ciudad de Puebla, en el sector comercial, que les ha permitido permanecer en el mercado en los últimos cinco años. La muestra fue a conveniencia, por consiguiente, se trata de una investigación no probabilística, integrado por 55 microempresas ubicadas en la zona comercial del centro de la ciudad de Puebla (compra-venta de artículos de consumo básico, como: mercerías, abarroteras, misceláneas, jugueterías y papelerías). Dichas empresas cumplen el requisito de una vida o existencia de 5 años o mayor. 


\section{Original}

PERCEPCIÓN DEL ÉXITO EMPRESARIAL, DESDE LA PERSPECTIVA DE PLANEACIÓN EN EL MICROEMPRESARIO EN PUEBLA.

Se aplicó una encuesta de forma presencial, con un instrumento de 22 preguntas. Como se muestra en la Tabla 1, el instrumento se operacionalizó y estuvo dividido en 2 grupos, 8 preguntas para identificar el perfil del microempresario y 14 preguntas para identificar los factores de éxito empresarial manejando una escala de Likert (del 1 al 5, siendo 1 totalmente en desacuerdo y 5 totalmente de acuerdo). Dicho cuestionario se validó por 3 expertos en gestión y desarrollo de organizaciones pertenecientes al Sistema Nacional de Investigadores del CONACYT y con una prueba piloto aplicada a 5 microempresarios, con el mismo perfil de la muestra.

\section{Tabla 1}

Operacionalización de variables

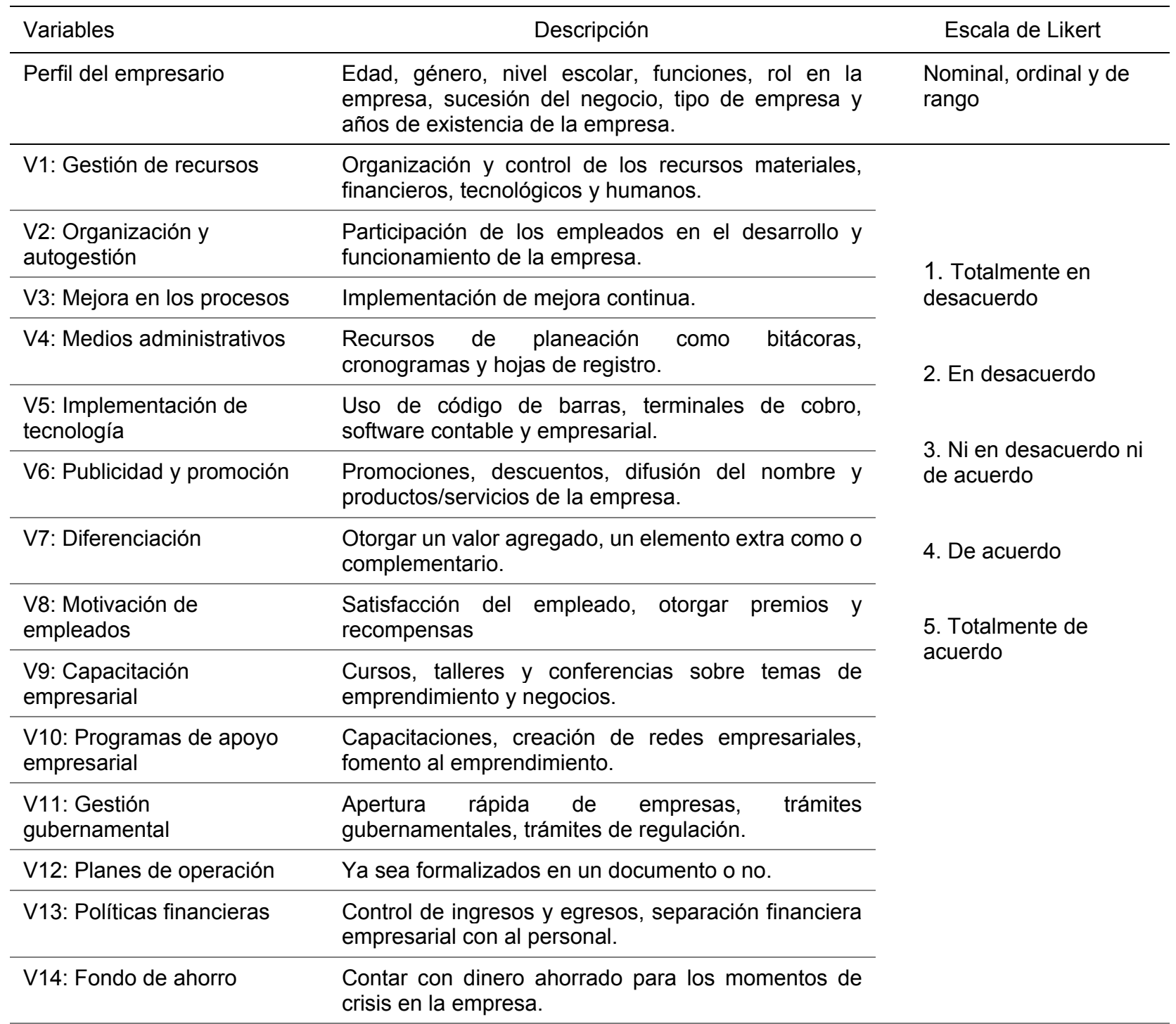

Fuente: Elaboración propia.

FLORES-BAUTISTA E. 
PERCEPCIÓN DEL ÉXITO EMPRESARIAL, DESDE LA PERSPECTIVA DE PLANEACIÓN EN EL MICROEMPRESARIO EN PUEBLA.

\section{ANÁLISIS Y DISCUSIÓN DE RESULTADOS}

Para medir la fiabilidad, como medida de confiabilidad de la investigación (Kerlinger, et al, 2002), se calculó el Alfa de Cronbach, resultando una fiabilidad aceptable de 0.782, de los 14 elementos (preguntas) de la variable factores de éxito empresarial, como se muestra en la Tabla 2 y de acuerdo a Kaplan \& Saccuzzo (2006, p. 106) "el valor de fiabilidad para la investigación básica entre 0.7 y 0.8 es aceptable".

\section{Tabla 2}

Fiabilidad de la investigación

$\frac{\text { Estadísticas de fiabilidad }}{\text { Alfa de Cronbach }}$
$\frac{0.782}{n}$

Fuente: Elaboración propia con SPSS v. 25

\section{Resultados: Perfil del empresario}

Con base en los datos analizados, el perfil de los empresarios del centro de la ciudad de Puebla, son jóvenes, debido a:

- Que el $19 \%$ tienen de 36 a 40 años, el $25 \%$ tienen de 31 a 35 años y el $22 \%$ tiene de 26 a 30 años; es decir, el $66 \%$ de los microempresarios tienen de 26 a 40 años.

- El $58 \%$ son mujeres y el $42 \%$ son hombre y en general, de todos ellos, el $22 \%$ solo tienen educación superior y el 78\% cuenta educación básica (sólo el 30\% cuenta con estudios de preparatoria).

Realizando un análisis detallado sobre este perfil del microempresario, el $47 \%$ de ellos son jóvenes entre 26 y 35 años, heredaron el negocio por parte de sus padres; es decir, el 53\% y sólo el $36 \%$ lo iniciaron con sus propios recursos y esfuerzos (ver figura 1). 
PERCEPCIÓN DEL ÉXITO EMPRESARIAL, DESDE LA PERSPECTIVA DE PLANEACIÓN EN EL MICROEMPRESARIO EN PUEBLA.

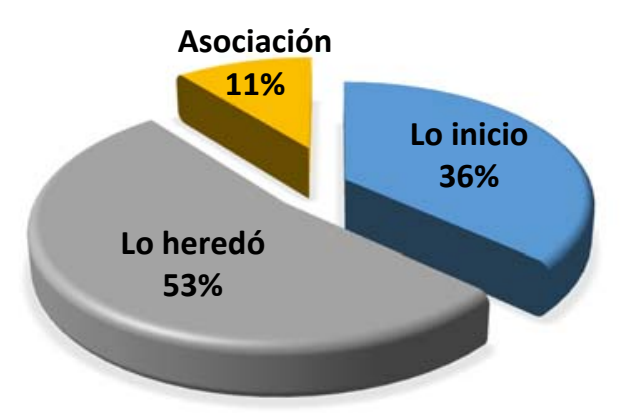

\section{Figura 1. Sucesión del negocio.}

Fuente: Elaboración propia con SPSS v25.

\section{Resultados: Análisis descriptivo}

Con base en los análisis de la encuesta aplicada a los microempresarios, en la zona comercial del centro de la Ciudad de Puebla, dedicados a la compra-venta de artículos de consumo básico como mercerías, abarroteras, misceláneas, jugueterías y papelerías, se tienen los siguientes factores de planeación y de éxito (según la percepción de los empresarios) para la permanencia comercial de sus empresas, con base en las experiencias de los últimos 5 años de vida del negocio. Dichas percepciones, son dadas por estos microempresarios:

- V1: Gestión de recursos. El 36\% de los microempresarios están totalmente de acuerdo (escala 5) y en de acuerdo (escala 4 ) un $23 \%$, que la gestión de recursos (organización y control de los recursos materiales, financieros, tecnológicos y humanos) dentro del negocio es un factor de éxito, con una media $\mu=3.71$, y una dispersión de los datos de $\sigma=$ 1.2; es decir, existe una variabilidad alta en la percepción, en el $59 \%$ de los microempresarios respecto a los recursos.

- V2: Organización y autogestión. El 31\% de los microempresarios están de acuerdo (escala 4) y en totalmente de acuerdo (escala 5) un 14\%, en que llevar a cabo organización interna y autogestión (participación de los empleados en el desarrollo y funcionamiento de la empresa) es un factor de éxito, con una media $\mu=3.74$, y una dispersión de los datos de $\sigma=1.04$; es decir, existe una variabilidad en la percepción, en el $45 \%$ de los microempresarios, respecto a la organización interna y la autogestión.

- V3: Mejora en los procesos. El 29.7\% de los microempresarios están de acuerdo (escala 4) y en totalmente de acuerdo (escala 5) un 19\%, en que mejorar los procesos (implementación de mejora continua) resulta como un factor para lograr el éxito, con una 
PERCEPCIÓN DEL ÉXITO EMPRESARIAL, DESDE LA PERSPECTIVA DE PLANEACIÓN EN EL MICROEMPRESARIO EN PUEBLA.

media $\mu=3.53$ y una dispersión de los datos de $\sigma=1.07$; es decir, existe una variabilidad en la percepción, en el $49 \%$ de los microempresarios, respecto a la mejora de los procesos.

- V4: Medios administrativos. El $36.7 \%$ de los microempresarios están totalmente de acuerdo (escala 5) y de acuerdo (escala 4) un 20\%, que los medios administrativos (recursos de planeación como bitácoras, cronogramas y hojas de registro) aplicados al negocio provocan el éxito del mismo, con una media $\mu=3.65$ y una dispersión de los datos de $\sigma=1.15$; es decir, existe una variabilidad en la percepción, en el $57 \%$ de los microempresarios, respecto al uso de medios administrativos.

- V5: Implementación de tecnología. El 35.7\% de los microempresarios están de acuerdo (escala 4) y totalmente de acuerdo (escala 5) el 14\%, en que la implementación de tecnología (uso de código de barras, terminales de cobro, software contable y empresarial) será un factor de éxito, con una media $\mu=3.69$ y una dispersión de los datos de $\sigma=1.06$; es decir, existe una variabilidad en la percepción, en el $50 \%$ de los microempresarios, respecto a la implementación de tecnología.

- V6: Publicidad y promoción. El 32.3\% de los microempresarios están de acuerdo (escala 4) y totalmente de acuerdo (escala 5) el 12\%, en que la publicidad y promoción (promociones, descuentos, difusión del nombre y productos/servicios de la empresa), es un factor que determina el éxito de un negocio, con una media $\mu=3.73$ y una dispersión de los datos de $\sigma=1.16$; es decir, existe una variabilidad en la percepción, en el $44 \%$ de los microempresarios, respecto al uso de publicidad.

- V7: Diferenciación. El $31 \%$ de los microempresarios está de acuerdo (escala 4) y totalmente de acuerdo (escala 5 ) el $21 \%$, en que el ofrecer un producto y/o servicio diferente (otorgar un valor agregado, un elemento extra como o complementario) ayuda al éxito de un micronegocio, con una media de $\mu=3.65$ y una dispersión de los datos de $\sigma=1.15$; es decir, existe una variabilidad en la percepción, en el $52 \%$ de los microempresarios, respecto a la diferenciación en el mercado.

- V8: Motivación de empleados. El 30.7\% de los microempresarios están de acuerdo (escala 4) y totalmente de acuerdo (escala 5) el 18\%, en que motivar a los empleados (satisfacción del empleado, otorgar premios y recompensas) ayuda al éxito del micronegocio, con una media de $\mu=3.61$ y una dispersión de los datos de $\sigma=1.24$; es decir, 
PERCEPCIÓN DEL ÉXITO EMPRESARIAL, DESDE LA PERSPECTIVA DE PLANEACIÓN EN EL MICROEMPRESARIO EN PUEBLA.

existe una variabilidad en la percepción, en el $49 \%$ de los microempresarios, respecto a la motivación del empleado.

- V9: Capacitación empresarial. El $31.7 \%$ de los microempresarios están de acuerdo (escala 4) y totalmente de acuerdo (escala 5) el 11\%, en que su capacitación empresarial (cursos, talleres y conferencias sobre temas de emprendimiento y negocios) para un micronegocio ayudará desarrollar mejores acciones empresariales para un mayor tiempo de permanencia en el mercado, con una media de $\mu=3.67$ y una dispersión de los datos de $\sigma=1.11$; es decir, existe una variabilidad en la percepción, en el $42 \%$ de los microempresarios, respecto a la capacitación empresarial.

- V10: Programas de apoyo empresarial. El 33\% de los microempresarios no están de acuerdo (escala 2) y totalmente en desacuerdo (escala 1) el $21 \%$, en que los programas de apoyo hacia los microempresarios (capacitaciones, creación de redes empresariales, fomento al emprendimiento) es un factor determinante de éxito, con una media de $\mu=2.50$ y una dispersión de los datos de $\sigma=0.89$; es decir, existe una variabilidad en la percepción, en el $54 \%$ de los microempresarios, respecto a los programas de apoyo empresarial.

- V11: Gestión gubernamental. El 33.7\% de los microempresarios están en desacuerdo (escala 2) y totalmente en desacuerdo (escala 1) el 14\%, en que buscar una gestión de apoyo del gobierno (apertura rápida de empresas, trámites gubernamentales, trámites de regulación) ayuda al éxito de los negocios, con una media de media $\mu=2.51 \mathrm{y}$ una dispersión de los datos de $\sigma=1.04$; es decir, existe una variabilidad en la percepción, en el $48 \%$ de los microempresarios, respecto a la gestión gubernamental.

- V12: Planes de operación. El 31\% de los microempresarios están de acuerdo (escala 4) y totalmente de acuerdo (escala 5 ) el 16\%, en que tener planes de operación a corto, mediano y largo plazo (ya sea formalizados en un documento o no) se convierte en factor para una mejor gestión del negocio, con una media de $\mu=3.72$ y una dispersión de los datos de $\sigma=1.14$; es decir, existe una variabilidad en la percepción, en el $47 \%$ de los microempresarios, respecto a contar con planes de acción.

- V13: Políticas financieras. El 28.3\% de los microempresarios están de acuerdo (escala 4) y totalmente de acuerdo (escala 5) el 13\%, en que manejar una política financiera (control de ingresos y egresos, separación financiera empresarial con al personal) ayudará al desarrollo exitoso de las actividades de la empresa, con una media de $\mu=3.57$ y una 
PERCEPCIÓN DEL ÉXITO EMPRESARIAL, DESDE LA PERSPECTIVA DE PLANEACIÓN EN EL MICROEMPRESARIO EN PUEBLA.

dispersión de los datos de $\sigma=1.14$; es decir, existe una variabilidad en la percepción, en el $41 \%$ de los microempresarios, respecto a las políticas financieras.

- V14: Fondo de ahorro. El 32.7\% de los microempresarios están de acuerdo (escala 4) y en totalmente de acuerdo (escala 5 ) el $16 \%$, en que resulta conveniente tener un fondo de ahorro (contar con dinero ahorrado para los momentos de crisis en la empresa) para cubrir problemas financieros inesperados a fin de mantener una estabilidad económica, con una media de $\mu=3.79$ y una dispersión de los datos de $\sigma=1.07$; es decir, existe una variabilidad en la percepción, en el $49 \%$ de los microempresarios, respecto a la existencia de fondos de ahorro.

\section{CONCLUSIONES}

Los microempresarios, en la zona comercial del centro de la ciudad de Puebla, dedicados a la compra-venta de artículos de consumo básico, como: mercerías, abarroteras, misceláneas, jugueterías y papelerías, consideran que el éxito de sus negocios se puede lograr a través de fondos de ahorro, con los cuales pueden capacitar a su personal, implementar tecnología e introducir productos nuevos. Asimismo, otros factores que son relevantes para estas personas son la implementación de tecnología, gestión de recursos, utilizar publicidad y propaganda. Además, para ofrecer un servicio o producto que se diferencie de lo demás, es necesario la motivación a los empleados, lo que puede diferenciarlos frente a la competencia. Algo importante de señalar, es la falta de confianza y credibilidad que tienen de las instituciones y programas de apoyo gubernamentales al emprendedurismo y el desarrollo empresarial, ya que sus experiencias ante estas no han sido del todo exitosas.

Se puede concluir, que los empresarios de los micronegocios han identificado inconscientemente los factores de éxito que han determinado la permanencia de su negocio, y a su vez los han aplicado sin tener un conocimiento profesional en algunos casos, mientras que otros, la falta de recursos provoca que sus ideas se vean sin impacto económico o de posicionamiento en sus negocios. Finalmente, todavía el empresario mexicano sigue viviendo un empirismo empresarial, pero con una creciente necesidad y claridad en formarse en estrategias de negocio, para lograr que sus empresas sobrevivan, permanezcan y sean rentables. 
PERCEPCIÓN DEL ÉXITO EMPRESARIAL, DESDE LA PERSPECTIVA DE PLANEACIÓN EN EL MICROEMPRESARIO EN PUEBLA.

\section{REFERENCIAS BIBLIOGRÁFICAS}

Arias Sandoval, A. \& Quiroga Marín, R. F. (2008). Cese de actividades de las pymes en el área metropolitana de Cali (2000-2004): un análisis de supervivencia empresarial. Cuadernos de Administración, 21(35).

Bautista, N. \& Benavides, J. (2013). Análisis de factores que afectan a las pymes en Colombia y herramientas financieras para controlarlas.

Chiavenato, I. (2011). Administración de recursos humanos: El capital humano de las organizaciones. México: McGraw-Hill.

Drucker, P. F. (2002). La gerencia en la sociedad futura. Editorial Norma.

Estrada, S. \& Dutrénit, G. (2009). Gestión del conocimiento en pymes y desempeño competitivo. un análisis comparativo de Querétaro y ciudad Juárez. G. Dutrénit, Sistemas Regionales de Innovación: un espacio para el desarrollo de las PYMES, el caso de la industria de maquinados industriales (pág. 317). México: Universidad Autónoma Metropolitana.

INEGI. (2014). inegi.org.mx. Obtenido de esperanza de vida de los negocios a nivel nacional y por entidad federativa: http://www.inegi.org.mx/inegi/contenidos/investigacion/Experimentales/Esperanza/doc/ev n_ent_fed.pdf

INEGI. (2015). www.inegi.org.mx. Obtenido de http://www.inegi.org.mx/est/contenidos/proyectos/encuestas/establecimientos/otras/enap roce/default_t.aspx

Kaplan, R. M. \& Saccuzzo, D. P. (2006). Pruebas psicológicas: principios, aplicaciones y temas. Thomson.

Kerlinger, F. N., Lee, H. B., Pineda, L. E. \& Mora Magaña, I. (2002). Investigación del comportamiento. México: McGraw-Hill.

Larios-Gomez, E. (2016a). La Gestión de la Competitividad en la MIPYME Mexicana: Diagnóstico Empírico desde la Gestión del Conocimiento. Revista de Administração da Unimep, 14(2).

Larios-Gomez, E. (2016b). La competitividad de mercadotecnia y la gestión del conocimiento en la MiPyME del sector retailing en Celaya, México. Revista de Administração, Contabilidade e Economia da Fundace, 8(1).

Martín, P. A. (2013). Factores que inciden en el éxito de las microempresas con mínimo cinco años de existencia del sector terciario del municipio de Arauca. Obtenido de https://core.ac.uk/download/pdf/12818556.pdf 


\section{Original}

PERCEPCIÓN DEL ÉXITO EMPRESARIAL, DESDE LA PERSPECTIVA DE PLANEACIÓN EN EL MICROEMPRESARIO EN PUEBLA.

Medina, M. O. \& de Lema, D. G. P. (2012). 40 factores explicativos del fracaso empresarial en las microempresas de la república dominicana. Título: Memorias Encuentro Internacional de Investigadores en Administración 2012 Compilado por: Alejandro Beltrán Duque, Augusto Rodríguez Orejuela y Carlos Alberto Restrepo Rivillas ISBN: 978-958-710-891-0, 661.

Moreno, F. M., Moreno, S. M. \& Dávalos, C. G. (2015). Planeación estratégica y gestión del conocimiento en las pequeñas y medianas empresas, (Pymes), herramienta básica para su permanencia y consolidación. European Scientific Journal, ESJ, 11(31).

Ortiz Useche, A., Rodríguez Monroy, C. \& Izquierdo, H. (2013). Gestión de mantenimiento en pymes industriales. Revista Venezolana de Gerencia, 18(61).

Parra, R. (2011). Plan Estratégico para Optimizar la Gestión de los Procesos Administrativos de las Pequeñas y Medianas Empresas (PYMES) familiares del Municipio Valencia del Estado Carabobo. Trabajo de Grado para optar al Título de Magíster en Administración de Empresas Mención Gerencia Postgrado de la Facultad de Ciencias Económicas y Sociales de la Universidad de Carabobo.

Pérez, M., Martínez, A., De Luis, P. \& Vela, M. J. (2006). Las TIC en las PYMES: Estudio de resultados y factores de adopción. Economía industrial, 360, 93-106.

Tello, C.; Hernández, C.; y Larios-Gómez, E. (2018). Contexto competitivo de la pyme en Puebla. Revista Ide@s Concyteg, Abril (184).

Tinoco-Cantillo, U. A., Buelvas-Martínez, A. M. \& Buelvas-Meza, C. G. (2016). Caracterización económica de las microempresas fabricantes de muebles de madera en la ciudad de Sincelejo. Perspectiva Socioeconómica, (4), 73-93. 